\title{
Anabases
}

ANABASES Traditions et réceptions de l'Antiquité

$9 \mid 2009$

Varia

\section{Entre archéologie, universalité et nationalismes : le trentième congrès eucharistique international de Carthage (1930)}

Jacques Alexandropoulos

\section{(2) OpenEdition}

Journals

Édition électronique

URL : http://journals.openedition.org/anabases/343

DOI : 10.4000/anabases.343

ISSN : 2256-9421

Éditeur

E.R.A.S.M.E.

\section{Édition imprimée}

Date de publication : 1 mars 2009

Pagination : 53-70

ISSN : 1774-4296

\section{Référence électronique}

Jacques Alexandropoulos, « Entre archéologie, universalité et nationalismes : le trentième congrès eucharistique international de Carthage (1930) », Anabases [En ligne], 9 | 2009, mis en ligne le 01 mars 2012, consulté le 20 octobre 2019. URL : http://journals.openedition.org/anabases/343 ; DOI :

10.4000 /anabases.343 
Anabases 9 (2009), p. 51-68.

\section{Entre archéologie, universalité et nationalismes: \\ le trentième congrès eucharistique international de Carthage (1930)}

JaCQues AleXandropoulos

Le CONGRÈS EuCHARISTIQUE DE CARTHAge, tenu en mai 1930, demeure généralement dans l'histoire contemporaine de la Tunisie comme un double symbole. Du côté du colonisateur, il fait partie des grandes célébrations de l'empire triomphant, au même titre que la commémoration du centenaire de l'Algérie, organisé la même année. Du côté du colonisé, il apparaît comme une provocation chrétienne en terre d'islam, l'effet d'une volonté d'évangélisation illustrée par d'autres signes, comme la statue du cardinal Lavigerie bénissant la foule, installée à l'entrée de la Médina de Tunis, au bas de la rue Djemaa-ez-zitouna qui mène à la mosquée du même nom. Certains aspects du congrès sont bien connus et ont été largement étudiés. C'est le cas par exemple de son effet désastreux sur l'opinion tunisienne. Et on date volontiers de ce congrès le véritable réveil des mouvements nationalistes qui devaient mener à l'indépendance ${ }^{1}$. On s'intéressera ici essentiellement à ses liens avec l'archéologie, l'histoire de l'Église d'Afrique et le jeu des nationalismes européens.

D'abord, qu'est-ce qu'un congrès eucharistique? "Une réunion d'hommes qui veulent ensemble étudier le dogme de l'Eucharistie, promouvoir son culte... faire mieux connaitre... et plus solennellement honorer Notre Seigneur dans le Saint-Sacrement ${ }^{2}$. " L'idée du premier congrès, tenu à Lille en 1880 revient à une pieuse lä̈que de Tours, Émilie Tamisier, encouragée par son confesseur, Pierre Julien Eymard, fondateur de la

1 Rapide survol par J.-F. Martin, Tunisie contemporaine, Paris, L'Harmattan, 1993, p. 94-95. Analyse des effets sur l'opinion tunisienne par A. MAHJOUBI, Origines du mouvement national, Publications de l'Université de Tunis, 1982, p. 466-479.

2 Lettre pastorale d'A. Lemaitre, archevêque de Carthage et primat d'Afrique, Actes et documents, Tunis, Édition de La Tunisie catholique, 1931, p. 8. 
congrégation du Très Saint Sacrement et surnommé "l'apôtre de l'Eucharistie ${ }^{3}$ ». Par la suite l'institution connut un succès croissant, avec des congrès prestigieux, comme celui de Jérusalem (1893), qui devinrent annuels à veille de la Première Guerre mondiale: Londres (1908) Cologne (1909), Montréal (1910), Madrid (1911), Vienne (1912), Malte (1913) et Lourdes (1914). Après la guerre ils reprirent en 1922 à Rome, 1926 à Chicago et 1928 à Sydney. À chaque fois, les cérémonies religieuses et les festivités réunirent des foules considérables venues de toute la catholicité - un record de deux millions de personnes à Chicago - avec des célébrations qui impressionnèrent les visiteurs, comme la procession du Saint-Sacrement dans la rade de Sydney ${ }^{4}$. Un rôle parfois politique de ces congrès est apparu, par exemple en 1938 (Budapest), lorsqu'Hitler interdit aux Allemands d'y participer, ou plus récemment en 1964 (Bombay) et 1968 (Bogota), lorsque Paul VI exhorta les pays riches au partage des richesses ${ }^{5}$.

Il s'agit donc au départ d'un rassemblement sans aucun lien particulier avec l'Afrique du Nord et son histoire ancienne. Alors pourquoi un congrès eucharistique à Carthage? La décision définitive en revient au pape Pie XI, mais l'idée en avait déjà été lancée depuis longtemps. Le P. Boubée, le jésuite qui fut la cheville ouvrière de l'organisation du congrès et le rédacteur des Actes $^{6}$, en attribue l'idée première à l'Académicien Louis Bertrand, le grand admirateur de l'Afrique romaine, en se référant à une page de son livre Le Sens de l'ennemi, qu'il qualifie de "page prophétique ${ }^{7}$ ». L. Bertrand, qui venait d'assister au congrès eucharistique de Lourdes (1914) imaginait une ville d'accueil pour l'un des suivants. Il pensa d'abord à Beyrouth, " un choix heureux car depuis l'époque des Croisades la vieille métropole syrienne n'aurait jamais vu pareille manifestation chrétienne. On renouerait solennellement une tradition. Mais, se dit-il, le fanatisme musulman est extrêmement susceptible. Ne serait-il pas imprudent d'aller l'inquiéter dans une ville où, somme toute, il est maître? " Il songea alors à Carthage: "Là rien à craindre des susceptibilités islamiques. Nous sommes chez nous, sur cette

Émilie Tamisier fut surnommée « la Jeanne d'Arc de l'Eucharistie » au Congrès de Madrid en 1911. Voir Le Nouvel Informateur Catholique, 11 juillet 2008: www.enlignetoi.com, Portail catholique et oecuménique d'information.

4 Carthage, 1930, Actes, p. 12;34-35.

5 La Croix, 14-15 juin 2008, p. 10.

6 La correspondance du P. Boubée, qui constitue avec l'imposant volume des Actes, l'essentiel de nos sources, est conservée aux Archives de la Prélature à Tunis. Ces dernières sont classées, mais sans posséder encore de numéros d'inventaire. Nous les citerons donc sans davantage de précision. Elles révèlent l'impressionnant travail accompli pour l'édition des Actes: "Mes supérieurs religieux m’ont envoyé ici sur la demande de S.G. Mgr Lemaître et du Comité permanent des Congrès internationaux pour essayer de réunir et de publier en volume les Actes du Congrès. Ce sera un travail d'autant plus long que je suis absolument seul, sans même un scribe ou une dactylo, et que les éléments sont dispersés à travers le monde " (lettre à $M^{\text {me }}$ de Castelnau du 28/10/1930, Archives de la Prélature). La correspondance du P. Boubée préparatoire au congrès témoigne de la même inlassable activité. Actes, p. 3. 
colline de Byrsa chantée par Virgile, baptisée par le sang de Félicité et Perpétue, illustrée par la mort de saint Louis, rachetée enfin par la France et par le Cardinal Lavigerie $^{8}$. " On retrouve ici un thème favori de L. Bertrand, celui de la vocation de la France à ressusciter l'Afrique latine et chrétienne, et qui lui valut d'être invité aux fêtes du congrès de Carthage où il fut un des orateurs les plus appréciés du public ${ }^{9}$. Mais d'autres raisons que cette perspective idéologique française ont sans doute joué dans le choix de ce lieu. D'abord, comme le fait remarquer l'archevêque de Carthage, Alexis Lemaître $^{10}$, relayé par la presse catholique, chaque continent avait eu son congrès, sauf l'Afrique. La loi d'œcuménicité demandait de réparer ce manque. L'année 1930, celle du jubilé des congrès eucharistiques dont le premier s'était tenu en 1880, en était une bonne occasion, et cela d'autant plus que 1930 correspondait aussi au quinzième centenaire de la mort de l'Africain saint Augustin fêté parallèlement à Bône-Hippone. Des liaisons spéciales entre Carthage et Bône furent d'ailleurs prévues pour permettre aux pèlerins d'assister aux deux festivités ${ }^{11}$. L'Afrique s'imposait donc aux yeux des autorités ecclésiastiques, et bien évidemment sur ce continent, Carthage, qui avait été le siège du Primat d'Afrique. Ce sont les principaux arguments qu'A. Lemaitre avait fait valoir auprès du Comité permanent des Congrès internationaux pour emporter une décision entérinée ensuite par le pape. On verra d'autres motivations plus loin.

Ce choix supposait l'ancrage du futur congrès dans une histoire et une tradition ecclésiologiques nord-africaine. Or cette histoire pouvait sembler particulièrement discontinue, semée de ruptures et de silences, l'avènement de l'islam étant évidemment la coupure principale. Les historiens suivaient la trace du christianisme africain antique jusqu'au XI $\mathrm{XI}^{\mathrm{e}}$ siècle dans la correspondance de la papauté avec la communauté chrétienne d'Afrique. Léon IX, dans deux lettres de 1053, évoque les cinq évêques qui restent in tota Africa, et Grégoire VII en 1076 s'adresse à l'évêque Cyriacus de Carthage comme au seul qui demeure en Afrique. Du côté de l'archéologie, des stèles funéraires datées des années 1007, 1019 et 1046, retrouvées à Kairouan, attestent encore sur place une communauté chrétienne organisée ${ }^{12}$. Tous ces éléments sont les dernières traces de la chrétienté de

L. Bertrand, Le Sens de l'ennemi, Paris, Fayard, 1917, p. 313.

Sur Louis Bertrand l'Africain, J. Dejeux, "De l'éternel Méditerranéen à l'éternel Jugurtha”, Studi magrebini 14 (1982), p. 70-89; J. Alexandropoulos, "De Louis Bertrand à Pierre Hubac: images de l'Afrique antique", in J. Alexandropoulos, P. Cabanel (éd.), La Tunisie mosä̈que, Toulouse, PUM, 2000, p. 457-478.

Avis officiel publié dans La Tunisie catholique du 25 décembre 1927, cf. Actes, p. 4.

Voir les lettres du P. Boubée à Mgr O'Gorman du 8 avril 1930 et à F. Veuillot, journaliste au Figaro, datée du 5 avril 1930. Cette dernière laisse toutefois entendre que le nombre des pèlerins sera modeste compte tenu du prix et des difficultés de transport (Arch. Prélature).

2 F. Decret, Le christianisme en Afrique du Nord antique, Paris, Seuil, 1996, p. 263-265; Voir aussi G. Lapeyre, J. Ferron, Carthage chrétienne, Paris, Letouzey et Ané, 1948, col. 61-64, qui évoquent deux allusions d'Ibn Khaldoun à des Berbères chrétiens au $\mathrm{XIV}^{\mathrm{e}}$ siècle. 
l'Afrique antique. Pour les historiens, c'est ensuite "l'islam et l'oubli ", " la fin d'un continent chrétien ${ }^{13}$ ». "La conquête almohade acheva de rappeler aux Maghrébins les rudes exigences de la foi. Avec Abd-el-Moumin les derniers chrétiens n'eurent le choix qu'entre la conversion et la mort ${ }^{14}$. " Pour les idéologues coloniaux, la présence française correspondait évidemment au grand retour de la chrétienté au Maghreb, mais le vide chronologique intermédiaire n'en demeurait pas moins gênant. Et Louis Bertrand s'employait à le combler à sa façon. D'abord en le minimisant par l'évocation lyrique et douloureuse d'une sorte de permanence de l'Antiquité. En visite à Carthage avec le coadjuteur de l'évêque il est saisi d'émotion, comme le prélat, au souvenir des saints et martyrs de l'Afrique antique et il leur semble les voir se dresser autour d'eux: "Ces misérables glorieux, ils ressuscitent, ils sont là autour de nous, avec les mêmes visages, les mêmes costumes qu'autrefois, ils poussent leurs ânes au flanc des collines pierreuses, ils piochent le sol avec leurs hoyaux primitifs, ou bien curieusement ils se penchent avec nous au bord de ces cuves baptismales, dont les inscriptions n'ont plus de sens pour eux, mais dont ils sentent confusément que c'est l'œuvre de leurs pères... Est-ce que tout est fini entre nous? Est-ce que ces âmes-là sont si loin des nôtres ${ }^{15}$ ? " L'autre manière de combler le hiatus était, on le sait, de minimiser la part arabo-musulmane médiévale et moderne de l'identité maghrébine comparée à un " décor " masquant une véritable identité latine. Le thème est trop connu pour que l'on y revienne ${ }^{16}$.

Cette image d'une rupture suivie, pour les idéologues, d'un légitime retour après des siècles d'islam, cette vision discontinue, est nuancée, lors de la préparation du Congrès eucharistique par une perception plus linéaire et continue de l'Église d'Afrique dans le temps, axée autour du martyre comme témoignage dans la souffrance, et de la croisade pacifique comme reconquête des âmes. En effet, la lettre apostolique par laquelle A. Lemaître annonce et présente le congrès, sans nier la "ruine " de l'Église d'Afrique à la fin de l'Antiquité avant sa " résurrection " contemporaine, insiste sur sa continuité entre temps et sur les "rayons de lumière " qui s'échappaient alors de sa tombe: "Durant les siècles douloureux qui suivirent sa ruine... elle ne cessa d'offrir chaque jour des brebis au couteau. " Il évoque ainsi les captifs "... enlevés par les Maures sur les côtes de France, d'Italie et d'Espagne " qui pouvaient faire, "dans l'intérieur des bagnes... où l'on portait la Sainte Hostie... des processions... suivies par une foule, dont les liens et les haillons lui faisaient, pour un regard chrétien, un magnifique triomphe ${ }^{17}$ ». Cette église médiévale et moderne rejoint à travers la souffrance la grande tradition des martyrs africains de l'Antiquité et met ainsi en évidence, outre

Y. Moderan, "La fin d'un continent chrétien”, Le Monde de la Bible, n 132, jan.-fév. 2001, p. 50.

L. Bertrand, Les Villes d'or, Paris, Fayard, 1921, p. 124-125.

Les villes d'or, p. 6.

Actes, p. 11. 
une continuité, une spécificité martyriale de l'Église d'Afrique qui sera l'un des thèmes essentiels du congrès. Cette spécificité ne marginalise pas pour autant cette église, bien au contraire. Lorsque l'on écrit dans $\mathrm{La}$ Croix à la veille du congrès qu' " après treize ou quatorze siècles de mort apparente, l'Église glorieuse de Carthage est ressuscitée ${ }^{18}$ ", on veut évidemment suggérer que l'histoire de cette Église, qui démarque par sa mort et sa résurrection l'événement de Pâques, devient un signe de portée universelle pour la chrétienté. Sa vocation martyriale donne par ailleurs à l'Église d'Afrique celle de témoin et de propagatrice exceptionnels de la foi chrétienne. Carthage devient alors non seulement le siège du plus ancien Primat d'Afrique, mais aussi le lieu qui ouvre sur tout un continent à évangéliser. Un prêtre breton, l'abbé Mévellec, désigné par son évêque avec d'autres, pour participer au congrès, nous a laissé un livre racontant ses souvenirs, et c'est bien ainsi qu'il voit les choses: « Devant ce golfe, les pèlerins du monde entier sentiront, comme un frémissement sacré, passer le souvenir des héros qui vécurent ou moururent ici pour la foi du Christ. En même temps, par delà les déserts $\mathrm{du}$ sud tunisien, ils croiront apercevoir les sables, les forêts les rivières, les montagnes du centre de l'Afrique, où pénétrèrent depuis un siècle, par tant de fissures glorieusement ouvertes, les rayonnements de cette foi chrétienne ${ }^{19}$. " Carthage est donc bien un lieu tout désigné pour le premier Congrès sur le continent africain.

Et la France peut aussi en tirer parti. En effet, après avoir évoqué dans sa lettre apostolique les captifs faits par les Maures sur les côtes de France, d'Italie et d'Espagne, A. Lemaître poursuivait ainsi: "Ces liens, sous lesquels ont gémi si longtemps les chrétiens de Tunis, sont aujourd'hui rompus. Sous l'égide de la France libératrice, il vous appartient de préparer à Notre-Seigneur un autre triomphe, plus magnifique que ceux d'autrefois, avec une procession où la sainte hostie sera suivie encore "par une foule", mais libre maintenant et en habit de fête, sous le regard émerveillé de ceux qui ne partagent pas notre foi ${ }^{20}$. "On saisit évidemment, à travers l'évocation de Mgr Lemaître, la dialectique tissée entre nationalisme et universalité catholique. Il rappelle d'une part la diversité d'origine des captifs, issus notamment des puissances rivales de la France au Maghreb, qui annonce la pluralité des participants au congrès (vingt-cinq nations représentées), et en regard l'identité de la nation libératrice, qui en est l'organisatrice en tant que maîtresse des lieux. Et dans cette perspective, la concomitance du congrès avec les fêtes du centenaire de l'Algérie française et le quasi cinquantenaire du protectorat en Tunisie ne pouvait être que bienvenue.

Au total, c'est donc sous la triple enseigne de l'Antiquité, de l'universalité catholique et du nationalisme que s'organise le congrès de Carthage. Comment se sont articulés ces trois éléments? Ils sont d'abord évidents dans le programme même du congrès.

$18 \quad$ La Croix du $1^{\mathrm{er}}$ avril 1930: Actes, p. 31.

19 Abbé F. MÉvelLeC, L’immortelle Carthage et les mystères du bled, Paris, Beauchesne, 1931, Avant-propos.

20 Actes, p. 11-12. 
Ce dernier était organisé sur cinq jours ${ }^{21}$. Le mercredi 7 mai était réservé à l'accueil, par les autorités françaises et tunisiennes du Protectorat ainsi que par l'archevêque de Carthage, des participants de marque, dont le légat du pape, le Cardinal Lépicier, avec les diverses délégations ecclésiastiques internationales. Tout cela sur la grande place du quartier européen de Tunis, la place de la Résidence, où se faisaient face la cathédrale et le palais de la Résidence générale de France, place bondée de monde et quadrillée par les troupes coloniales.

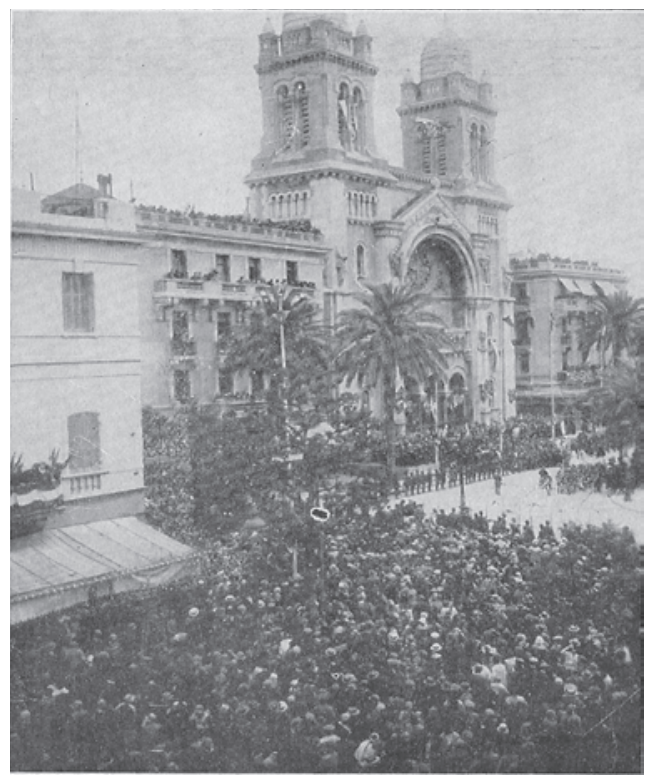

La place de la Résidence

lors de l'ouverture du congrès

(toutes les photos sont extraites

du volume des Actes)

La mission pontificale. Assis au premier plan : à gauche, le légat pontifical, le cardinal Lépicier ; à droite,

l'Archevêque, Alexis Lemaître

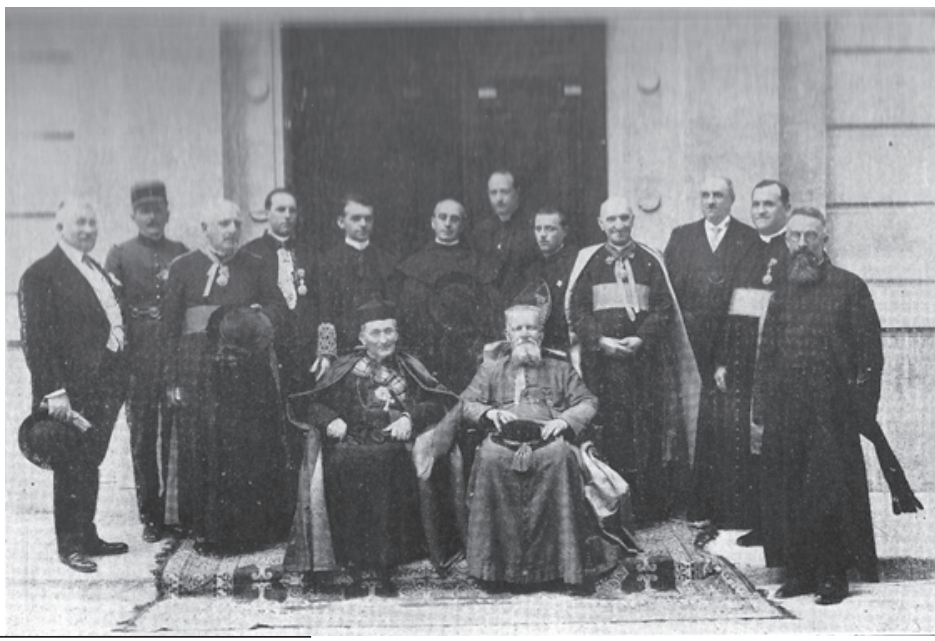
Actes, p. 69-75. 
Le jeudi 8 mai était réservé aux enfants, avec en clôture le défilé de six mille garçons et filles habillés en croisés, déposant des palmes à l'amphithéâtre romain de Carthage en l'honneur des martyrs chrétiens. Les trois derniers jours, jusqu'au dimanche 11 mai, s'ouvraient par des messes pontificales sur trois sites archéologiques chrétiens, successivement: la basilica maiorum, l'amphithéâtre romain et la basilique de saint Cyprien. Il y avait ensuite des réunions de travail théologiques par sections nationales réparties dans les diverses églises ou bâtiments de Tunis, avec des interventions de dignitaires ecclésiastiques en plusieurs langues. Ces journées étaient elles-mêmes clôturées par des assemblées générales. Celle du vendredi comprenait une conférence du vicaire général sur l'Eucharistie, suivie d'une retraite aux flambeaux, la " veillée des hommes ", à l'amphithéâtre de Carthage. Celle du samedi prévoyait le discours le plus attendu, l'évocation, par Louis Bertrand, de l'Afrique romaine et chrétienne, devant la basilique primatiale érigée sur le lieu de la mort de saint Louis. Le dernier jour était réservé aux cérémonies de clôture avec une bénédiction générale par le légat devant cette même Primatiale. L'archéologie et l'histoire structuraient donc fortement l'espace, le temps et la thématique du congrès, faisant de Carthage à la fois le lieu de mémoire et le lieu de résurrection de l'Afrique romaine chrétienne.

La préparation d'un tel congrès dans une capitale relativement petite, aux infrastructures routières limitées et aux capacités d'accueil réduites fut très difficile et met d'autant plus en relief le retentissement international que l'on voulait donner à cette résurrection de l'Afrique chrétienne sous l'égide de la France. Et si la réussite technique fit manifestement la fierté des organisateurs, le coût financier d'une manifestation religieuse chrétienne d'envergure internationale imposée par les autorités souleva l'indignation des opposants, socialistes laïques, dignitaires musulmans et nationalistes tunisiens ${ }^{22}$. Les services des travaux publics étaient sur les dents pour l'aménagement des lieux de congrès. Leur directeur en témoigne: "Carthage n'a jamais vu, depuis les Romains, s'agiter sur son sol une armée d'ouvriers comme celle qui y travaille depuis plusieurs mois. De toutes parts ce sont des ruines qu'on déblaie, des terrains qu'on nivelle, des routes qu'on empierre, des constructions qu'on élève. " Les voies d'accès

Ainsi, La voix du Tunisien du 10 mai 1930, titre : « Le congrès eucharistique... Une folie... La laïcité au vestiaire » et poursuit: «Qui ne voit là toute une cascade de provocations et de défis à la conscience de deux millions de musulmans? „; Tunis socialiste tonne contre l'obligation faite aux «neutres de se réfugier chez eux ou de se courber devant l'eucharistie » (6 mai 1930) et pastiche les recommandations aux pèlerins de La Tunisie catholique et les instructions des autorités: « Article VI: du 8 au 11 mai toute perturbation atmosphérique est interdite. Les rassemblements de nuages seront poursuivis conformément au décret beylical qui punit les délits de coalition » (10 mai 30). La ligue des Droits de l'Homme, pour sa part, écrit au ministère des Affaires étrangères que «l'opinion française, attachée aux principes de laïcité, et l'opinion tunisienne, attachée à la foi musulmane, sont d'accord pour regretter que les représentants de la France se soient départis, en cette circonstance, de la stricte neutralité religieuse qui est la base de nos institutions » (cité par La Croix du 14 juin 1930). 
sont réaménagées, la route de Tunis à Carthage est élargie « jusqu’à devenir une magnifique autostrade 23 ». Tous les habitants, catholiques ou non, furent sollicités pour l'hébergement des pèlerins. L'armée organisa de grands camps de toile à Carthage avec cantines et églises de campagne pour loger l'essentiel des participants.

La circulation des voitures était partout canalisée. La presse accompagnait quotidiennement et chaudement l'événement, pour aider à l'organisation, informer, s'extasier ou au contraire s'indigner. Le mouvement dépassait Tunis et c'est toute la Régence qui vivait au rythme du congrès, ne serait-ce que par les déplacements touristiques des congressistes $^{24}$.

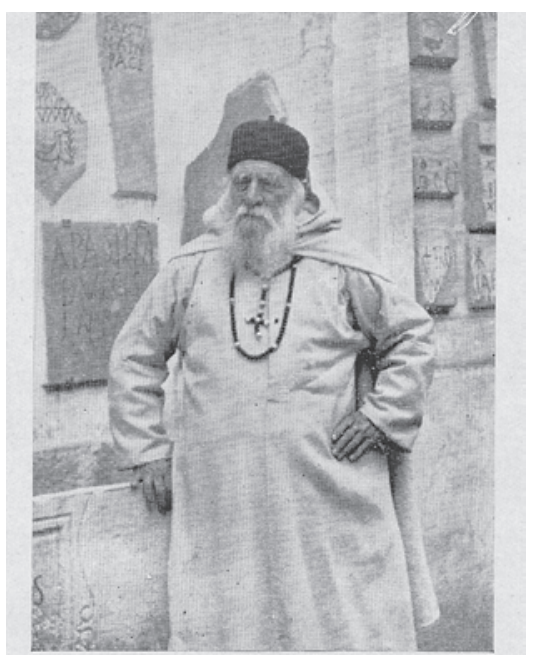

Une des dernières photos du P. Delattre

Mais parmi tous ces préparatifs, le côté archéologique tenait évidemment une place essentielle. En ce sens, le congrès marque une sorte d'aboutissement. Les fouilles de Carthage avaient été conçues dès l'origine comme un élément important de l'action des missionnaires de Lavigerie. En 1875 le Cardinal les envoyait à Carthage avec mission d'y soigner et enseigner les pauvres, mais, disait-il dans une lettre à l'Académie, si ces missionnaires "ne pensaient qu'à éclairer les barbares de l'Afrique... je ne crois pas les détourner de leur œuvre en les chargeant de prouver, en outre, par des faits, aux civilisés de notre Europe, que l'Église n'a pas cessé d'être l'amie de la science ${ }^{25}$ ». Et les instructions données aux Pères Blancs étaient nettes: "Votre étude constante devra être partagée entre l'arabe et l'archéologie carthaginoise, sacrée et profane ${ }^{26}$. " Toute cette activité, accompagnée de la construction d'un dispensaire et d'un collège à Carthage, s'intégrait dans l'une des préoccupations principales de Lavigerie: obtenir la restauration d'un siège épiscopal à Carthage, consacrant la résurrection de l'Église d'Afrique. Il n'existait depuis 1843 qu'un vicariat apostolique qui faisait lui-même suite aux missions des Capucins, puis des Lazaristes envoyés depuis le

Actes, p. 55. On pense évidemment en filigrane à l'évocation virgilienne de la construction de Carthage par Didon (Enéide, I, 420-437), allusion, elle-même au gigantesque chantier de reconstruction augustéenne.

24 Voir le périple de MÉvellec, L’immortelle Carthage, p. 89-150, avec une description de l'engorgement des structures hôtelières de Sousse, p. 121-123.

25 A.L. Delattre et G. Lapeyre, L'archéologie et le Congrès eucharistique de Carthage, Tunis, Aloccio, 1932, p. III-IV.

26 F. Renault, Le Cardinal Lavigerie, Paris, Fayard, 1992, p. 439. 
$\mathrm{XVII}^{\mathrm{e}}$ siècle auprès des captifs chrétiens ${ }^{27}$. La formule instauranda est Carthago résumait cette volonté de voir revenir un évêque à Carthage - ce sera fait en 1884 -, et la construction terminée en 1890 d'une basilique primatiale sur la colline saint Louis, dont la première pierre venait de l'antique basilique chrétienne de Damous El Karita, devait en être l'aboutissement monumental. Dans cette perspective à long terme de résurrection de Carthage, la recherche archéologique, principalement chrétienne, était évidemment un élément indispensable. C'est elle qui allait justifier un congrès eucharistique à Carthage et ce dernier lui offrait en retour un couronnement international. Au point que le $P$. Delattre pouvait écrire juste après le congrès: « Ce sont les découvertes archéologiques, qui, dirigées pendant plus d'un demi-siècle par la Providence, devaient préparer le terrain et rendre possible la célébration du Xxx congrès eucharistique international ${ }^{28}$. » Si la Providence avait dirigé les travaux, le P. Delattre s'en disait «l'humble ouvrier ». Envoyé à Carthage en 1875, un an après son engagement définitif chez les missionnaires d'Afrique, il avait régné en maitre sur le site, montrant une activité d'archéologue infatigable, publiant régulièrement en lien avec l'Académie le résultat de ses découvertes, et pour lui aussi le congrès marquait la fin d'une carrière. Durant les cérémonies de la fête des enfants à l'amphithéâtre, il fut ovationné: "Le P. Delattre, l'infatigable chercheur de Carthage, devant le tonnerre d'applaudissements qui accueille son entrée, refuse d'avancer plus loin, et va cacher sa modestie dans la foule anonyme ${ }^{29}$. " Après le congrès, l'archevêque lui rend un hommage appuyé: "Le Congrès Eucharistique International de Carthage a resplendi, devant vingt-cinq Nations représentées, d'un éclat inoubliable. A-t-on suffisamment réfléchi, à ce que le Congrès... eût laissé de vague sans vos travaux. Et, au contraire, à ce que ces travaux de cinquante ans, les vôtres, ont procuré de poignantes émotions à l'âme de ces milliers de fidèles, de ces milliers de prêtres et de futurs prêtres, quand ils pouvaient se dire: c'est bien ici, sur cet amphithéâtre, qu'ils ont vécu, qu'ils ont lutté et donné leur vie pour le $\mathrm{Christ}^{30}$. » Et ces remerciements arrivent juste avant le décès du P. Delattre, le 11 janvier 1932, un an et demi après la clôture du congrès. Comme le soulignait A. Lemaitre, cette manifestation venait véritablement mettre un point d'orgue à un demi-siècle de travaux, d'études et de remise à l'honneur de l'Afrique chrétienne, et elle créait ainsi l'événement qui consacrait Carthage comme lieu de mémoire chrétien.

On pouvait retrouver cette présence active de l'archéologie, lors des cérémonies, sur trois plans: dans le paysage archéologique apprêté pour l'occasion, dans les images et les objets créés et utilisés pour les cérémonies, et dans les chants, prières et discours des participants. L'organisation du paysage archéologique repose d'abord sur des monuments antiques mis à l'honneur: d'une part l'amphithéâtre où furent martyrisés

Renault, Le Cardinal Lavigerie, p. 424 sqq.

L'archéologie, p. 3.

Mévellec, L'immortelle Carthage, p. 171.

30 A. Lemaitre, 7 novembre 1931; DelatTRe, L'archéologie, introduction. 
les chrétiens, d'autre part les trois principales basiliques de Carthage retrouvées à cette époque. L'amphithéâtre, sur un terrain racheté par Lavigerie et fouillé par Delattre, était extrêmement ruiné, réduit à une arène quasiment sans gradins, mais sous laquelle subsistait un couloir souterrain aboutissant à une salle voûtée. Dès avant le congrès, cette salle avait été transformée en chapelle dédiée aux saintes Perpétue et Félicité, avec un autel dont les colonnes de marbre venaient de la basilique de Damous El Karita. Une plaque rappelait qu'" ici (avaient été) martyrisées le 7 mars de l'an 203 sainte Perpétue et sainte Félicité, exposées à la dent des bêtes avec $S$. Révocatus, S. Saturus et S. Saturninus ". Cet amphithéâtre allait devenir l'un des hauts lieux du congrès pour accueillir l'offrande des palmes par les petits croisés, puis le soir suivant, la retraite aux flambeaux des hommes, le tout sous la haute présidence du nonce apostolique.

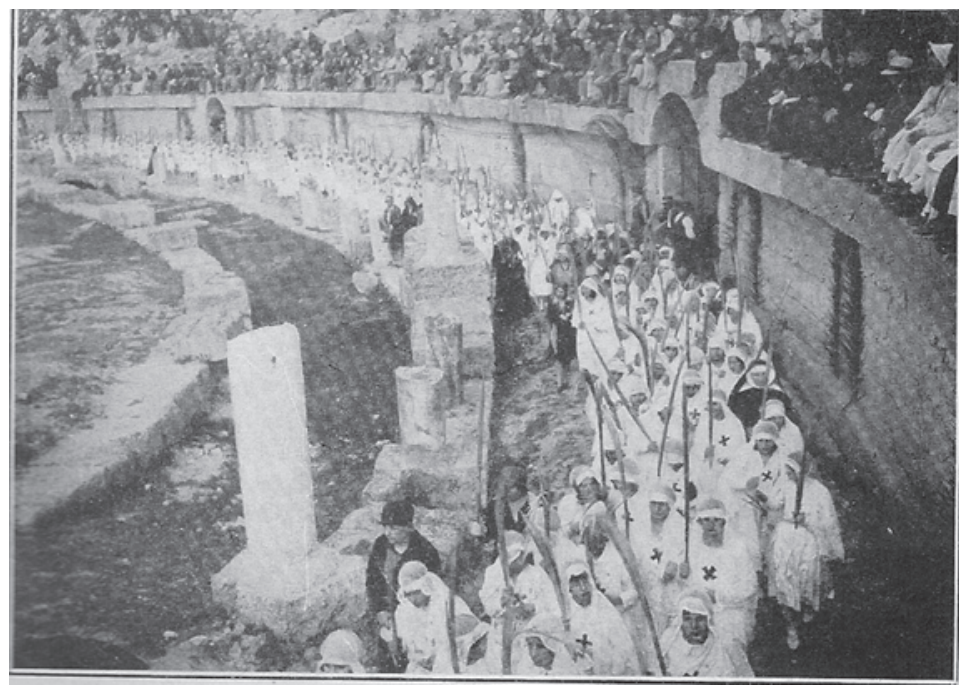

Le défilé des petits croisés dans l'amphithéâtre

Dès lors il avait été question de reconstruire les gradins pour l'occasion et on avait même dit que l'archevêque rebâtirait tout le monument... en bois il est vrai ${ }^{31}$. En fait, on se contenta de monter une immense estrade au fond de l'arène, supportant le siège du nonce abrité d'un dais et tous ceux des prélats. Une grande fresque à thème martyrial couvrait tout le mur du fond de l'arène. Quatre mâts portaient des étendards avec l'inscription constantinienne IN HOC SIGNO VINCES et au centre flottait un drapeau aux couleurs du pape. L'amphithéâtre était devenu « une splendide cathédrale à ciel ouvert ${ }^{32}$ ».

Trois basiliques servirent aussi d'une manière ou d'une autre aux festivités. La plus grande et la plus ancienne trouvée par le P. Delattre, celle de Damous El Karita, trop excentrée sans doute, et surtout impossible à identifier et à relier à un contexte antique connu ou à un événement important du christianisme antique, fut laissée de

\footnotetext{
31 Delattre, L'archéologie, p. 6-19.

32 Delattre, L'archéologie, p. 17 (mot du P. Parra).
} 
côté. Néanmoins on y célébra quelques messes, et surtout, un fragment de sculpture antique qui y avait été recueilli servit de base à l'élaboration pour l'occasion d'une effigie de Notre Dame de Carthage, imprimée à Toulouse à huit cent mille exemplaires vite épuisés et reproduits, et frappée en médaille commémorative du congrès. Au total ce seraient plusieurs millions de reproductions qui auraient été diffusées d'une manière ou d'une autre ${ }^{33}$. La deuxième basilique est la Basilica maiorum où un texte de Victor de Vita disait que les corps des martyres Perpétue et Félicité avaient été enterrés ${ }^{34}$. Or, en 1907, sur un terrain difficilement acheté par le P. Delattre qui y retrouvait d'innombrables petits fragments d'inscriptions funéraires chrétiennes, on avait pu finalement récupérer et rassembler le véritable puzzle de l'inscription funéraire des deux martyres. Le site semblait donc identifié comme étant la Basilica maiorum et les autorités religieuses de Tunisie, à l'initiative d'une congrégation qui honorait particulièrement les deux saintes, obtinrent dès lors du Vatican, en 1909, qu'une fête en leur honneur soit inscrite au calendrier religieux. Jusqu'au congrès l'endroit était très peu visité, mais peu avant, le P. Delattre y fit construire une petite chapelle souterraine appelée " confession ", surmontée d'un " ciborium ", baldaquin en ciment armé décoré de marbre et supporté par quatre colonnes. On érigea à côté une statue de saint Augustin qui avait souvent prêché sur place. La statue de l'évêque avec mitre et crosse, ayant à ses pieds l'Évangile surmonté de l'aigle de saint Jean était une réplique de celle d'Hippone. Pour le congrès on releva une partie des murs extérieurs, on installa de nombreux oriflammes aux couleurs des dignitaires ecclésiastiques, ou représentant des scènes martyriales. Ce fut le lieu de nombreuses messes dont la messe pontificale célébrée par l'évêque de Cagliari ${ }^{35}$. Mais la plus utilisée des basiliques fut celle de saint Cyprien. Découverte par le P. Delattre durant la Première Guerre mondiale, elle présentait un plan intact à sept nefs et on en avait relevé les murs extérieurs et une partie des colonnes.

Elle avait par ailleurs une forte valeur symbolique: son emplacement correspondait au lieu d'ensevelissement de saint Cyprien et ce pouvait être de là que saint Augustin s'était embarqué pour Rome au désespoir de sa mère, Monique. Depuis 1921 la basilique servait aux processions du Saint Sacrement organisées par le couvent voisin de sainte Monique: "Qui aurait songé alors que tout cela n'était que le prélude des grandioses manifestations du Congrès Eucharistique? » s'exclame le P. Delattre. Ce fut le lieu choisi pour la messe de clôture du congrès. C'est alors qu'on y édifia pour l'occasion le baldaquin d'après des modèles byzantins, et une immense estrade pour tous les dignitaires. "C'est vers l'autel du Saint Sacrifice que se dirigeaient les regards de milliers et de milliers de personnes ex omni tribu, et lingua, et populo, et natione. La foule...

33 Delattre, L'archéologie, p. 20-24.

34 Sur tous ces monuments chrétiens de Carthage, voir l'étude archéologique récente de L. Ennabli, Carthage. Une métropole chrétienne du Ive à la fin du VII siècle, Paris, CNRS, 1997.

35 Delattre, L'archéologie, p. 25-44. 
couvrait tout le plateau de Sainte-Monique, les collines de Carthage, la falaise de Sidi Bou Saïd et tous les points... d'où l'on pouvait apercevoir le Cardinal-Légat pontifiant. Très impressionnants, les instants de complet silence dans cette immense foule, au moment de la consécration et de l'élévation. Spectacle inoubliable, défiant toute description complète. Jamais l'Église de Carthage, même au temps de sa splendeur, n'assista à de pareilles cérémonies. Cela surpassait de bien loin ce que saint Augustin avait pu voir de son vivant ${ }^{36}$. " L'Église d'Afrique semblait effectivement renaître et consacrer ainsi l'ensemble d'une œuvre archéologique chrétienne.

Le lien tissé par l'archéologie entre passé et présent allait plus loin que la mise en œuvre des monuments antiques restaurés et aménagés pour l'occasion. La transformation du site archéologique en lieu de mémoire supposait de combler les manques et, sur la route qui gravissait la colline de Carthage dominée par la primatiale, on bâtit en carton et bois un arc de triomphe, réplique de celui du site de Sbeïtla, de treize mètres de haut sur douze de large. Outre sa valeur triomphale, il était pour les congressistes venus par le train, la porte d'entrée dans Carthage. L'inscription de l'arc originel, érigé en l'honneur des tétrarques persécuteurs Dioclétien et Galère avait été remplacée par une inscription en latin: "O notre bienheureuse Carthage, éclatante de blancheur par

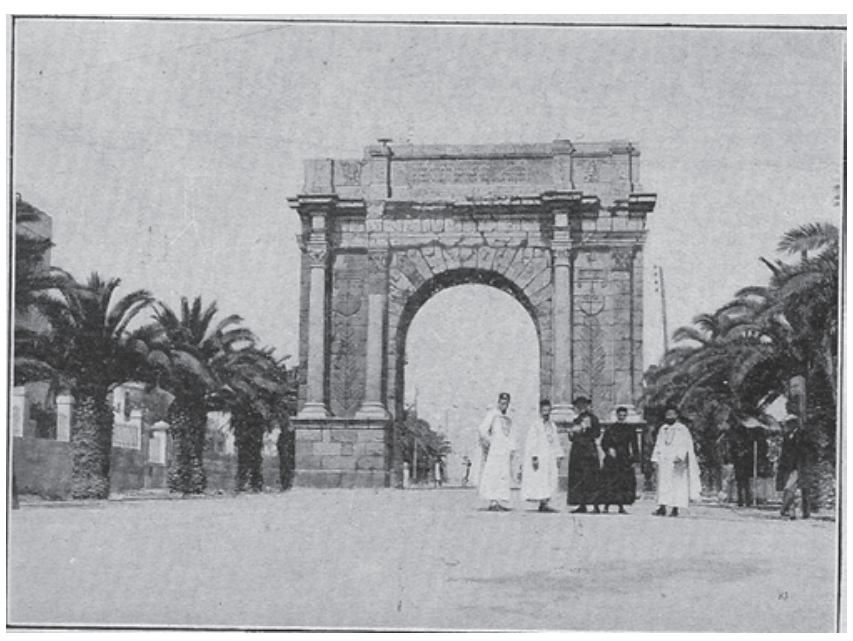

Réplique de l'arc de Sbeïtla érigée à Carthage pour le congrès la pureté des œuvres de ses enfants; elle est maintenant empourprée du sang de ses martyrs; elle a aussi, parmi ses fleurs des lys et des roses. " On y trouvait aussi, outre une représentation du Bon Pasteur reprise d'une... des catacombes d'Hadrumète, la formule célèbre de saint Cyprien: sanguis martyrum semen christianorum, qui était par ailleurs le titre d'un ouvrage célèbre de L. Bertrand sur les martyrs d'Afrique ${ }^{37}$. C'est sous cet arc que devaient passer le défilé des enfants croisés et la procession du Saint Sacrement en clôture du congrès.

Le point culminant du site, dans tous les sens du terme, était évidemment la Primatiale elle-même, érigée entre 1884 - date de la création de l'évêché de Carthage 
- et 1890 par Lavigerie. Elle consacrait la résurrection de l'Église d'Afrique et ses liens avec l'Antiquité étaient donc nombreux. Sa situation au point le plus haut de Carthage en faisait le résumé et le point d'aboutissement de l'histoire du site, lui donnait son sens ultime: c'est au même endroit qu'aurait été bâti le temple d'Eschmoun, dernier lieu de résistance de la Carthage punique, que les arpenteurs romains auraient situé le point d'origine de leur cadastration lors de la refondation du site, que le P. Delattre imaginait le Capitole, et qu'était mort, pensait-on, saint Louis. Elle était placée sous l'invocation de deux martyrs: saint Cyprien et saint Louis. À l'intérieur une inscription rappelait le message de Léon IX aux chrétiens d'Afrique en 1054: "Sans aucun doute, après le Pontife romain, le premier Archevêque et suprême métropolite de toute l'Afrique, c'est l'évêque de Carthage. Pour aucun autre Evêque d'Afrique, quel qu'il soit, celui-ci ne peut perdre le privilège qu'il a reçu une fois pour toutes du Saint-Siège apostolique et romain. Il le conservera jusqu'à la fin des siècles, tant que sur cette terre d'Afrique on invoquera le nom de Notre Seigneur Jésus-Christ, soit que Carthage reste gisante et en ruines, soit qu'elle connaisse un jour la gloire d'une résurrection. " La visite type des congressistes comportait donc le passage par la porte triomphale, la visite de l'amphithéâtre, des trois basiliques principales et enfin de la Primatiale, couronnement et sens ultime de l'ensemble du lieu de mémoire ${ }^{38}$.

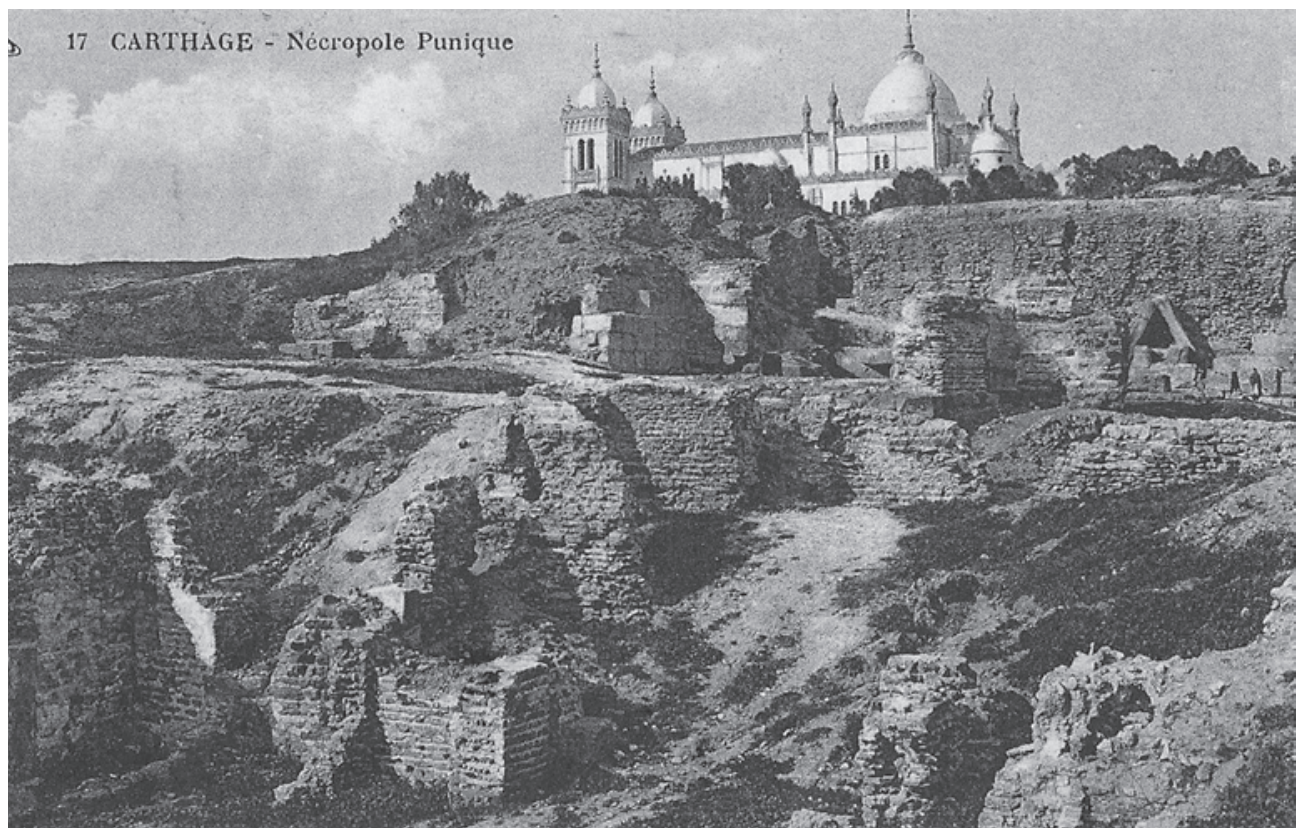

Vue des vestiges puniques et romains de Byrsa, surmontés par la Primatiale

38 Pour la Primatiale, voir les impressions de MÉvellec, L’immortelle Carthage, p. 49-52. Il y avait d'autres lieux de visite importants, comme l'ager sextius où saint Cyprien avait été exécuté et l'ensemble des couvents et chapelles de Carthage: Delattre, L'archéologie, p. 73. 
La double thématique du martyre et de la résurrection qui sous-tendait le paysage du congrès, et que l'archéologie avait construite au fil du temps par l'intermédiaire de "l'ouvrier de la Providence " qu'était le P. Delattre, trouvait un écho dans les chants, les discours et le rituel qui accompagnèrent les pèlerins. Outre les chants religieux traditionnels, trois autres avaient été composés pour l'occasion: la cantate officielle du congrès, choisie après un concours de trois cent cinquante participants internationaux, le cantique de l'offrande des palmes et l'invocation à Carthage. Les trois étaient entièrement dominés par le thème du martyre des chrétiens d'Afrique - celui-ci revenant de manière véritablement obsédante - de la mort de saint Louis et celui de la catholicité, au sens d'universalité, des participants au congrès ${ }^{39}$. Les objets même, comme l'ostensoir, avaient été reliés à l'Antiquité, puisque ce dernier reprenait les thèmes d'une mosaïque qui aurait orné une basilique élevée sur les lieux de l'exécution de saint Cyprien: un calice reposant sur un rocher d'où coulent quatre sources auxquelles viennent boire un cerf et une biche ${ }^{40}$. Quant au rituel il comprenait une litanie des saints et martyrs d'Afrique composée par Lavigerie lui-même. Les discours, eux, se partageaient entre une réflexion sur l'Eucharistie, thème des Congrès en général, et la célébration de l'antique Église d'Afrique. On retrouvait en outre dans celui de L. Bertrand un vibrant éloge de l'œuvre archéologique accomplie. Ce discours était le plus attendu, comme nous le dit l'abbé Mévellec qui s'extasiait de tout:

Après dîner, chacun organise son après-midi. Pourvu que nous soyons tous présents à 5 heures, sur la colline de Carthage, pour le grand discours de Louis Bertrand. D'ores et déjà on en parle comme devant être le clou de la fête... Voici maintenant Louis Bertrand, en habit d'académicien. Il est venu là précédé d'une grande réputation, et tous ceux qui ont suivi la marche de son travail d'Africain ardent et convaincu, ont tenu à se trouver ce soir à l'assemblée générale... Et Louis Bertrand, calme et grave comme un vieux Latin, se pose devant le microphone. Il commence dans un silence religieux... Louis Bertrand est un grand classique. Son génie est fait de l'alliance d'une forte imagination et d'un ferme jugement. Dans ses tableaux, le tracé est net et les couleurs sont vives. Peu à peu l'émotion nous gagne, nous pénètre, et c'est les larmes aux yeux que nous écoutons maintenant l'histoire de la chrétienté africaine ${ }^{41}$.

On remarquera que cet engouement pour L. Bertrand fait suite à un temps de méfiance que reflète un échange de lettres entre l'archevêché de Tunis et divers prélats de France. On s'était en effet efforcé, juste avant le congrès, de régler un point délicat: on avait d'emblée invité $L$. Bertrand à y participer, en oubliant que son ancienne appartenance à l'Action Française le rendait personna non grata aux yeux du Vatican. Mais, 
discrètement rassuré, A. Lemaître avait pu maintenir l'invitation ${ }^{42}$. Néanmoins, le nonce apostolique se fit excuser au dernier moment pour le discours de l'Académicien: il était retenu au palais beylical par une audience accordée aux petites filles du Bey ${ }^{43}$.

La conclusion sur l'interaction de l'archéologie et du congrès nous est fournie par le P. Delattre lui-même:

Il est permis de dire que l'archéologie a joué un rôle important dans la préparation lointaine et prochaine, ainsi que dans la réalisation du Congrès Eucharistique de Carthage. On ne peut donc qu'admirer combien le Cardinal Lavigerie fut bien inspiré en créant ici une œuvre de recherches scientifiques qui devait tant faire honneur à l'Église, au Diocèse de Carthage et à la Société des Pères Blancs, en contribuant un jour à la possibilité et au succès du XXX ${ }^{\mathrm{e}}$ congrès Eucharistique International ${ }^{44}$.

Le congrès apparait bien comme le triomphe de l'archéologie religieuse, dans tous les sens du terme, en Tunisie.

De quelle manière le congrès servait-il du même coup le nationalisme français? Les discours officiels ne montrent pas de récupération directe et explicite au profit de la France. La lettre de vœux de plein succès envoyée par le Pape ne fait aucune allusion aux puissances invitantes ${ }^{45}$. Lors de la cérémonie d'ouverture, l'allocution de l'évêque de Namur, Mgr Heylen, président du comité d'organisation, ne comporte qu'un discret remerciement de quelques mots au Bey et au Résident dont le titre apparaît incomplet et dont le nom n'est pas prononcé, non plus que celui de la France ${ }^{46}$. Le discours du Cardinal-Légat, Mgr Lépicier, ne mentionne, lui non plus, aucune autorité ${ }^{47}$. En fait, l'hommage aux autorités civiles et militaires françaises se fait très cordialement mais très discrètement à la Résidence Générale, au Tunisia Palace, lors d'un banquet offert par A. Lemaître, et lors d'une fête militaire. Les Actes du congrès le montrent: bien que le protocole ait été respecté, il n'y eut que des échanges informels, sans discours prononcés, rien que des conversations très libres et très cordiales ${ }^{48}$. En revanche il y eut plusieurs visites officielles et audiences chez le Bey et sa famille, et parmi les audiences du Légat remarquées il y eut celle du Prince Taïeb, fils du Bey et celle du rabbin-doyen de la communauté juive livournaise, Yacoub Boccara ${ }^{49}$. En somme, on respectait la fiction politique du Protectorat et l'accent était continuellement mis sur l'aspect international du congrès. La récupération nationaliste est pourtant évidente dans la mesure

Voir, aux Archives de la Prélature, la correspondance entre le P. Boubée et divers prélats, dont Mgr Gerlier et Mgr Crépin, entre octobre et décembre 1929.

MÉvelLeC, L'immortelle Carthage, p. 203.

Delattre, L'Archéologie, p. 72.

Actes, p. 102-103.

Actes, p. 103-104.

Actes, p. 104-106.

Actes, p. 107.

Actes, p. 109-110. 
où on vérifie une nouvelle fois que la laïcité n'est pas un article d'exportation pour les colonies. La présence des plus hautes autorités civiles et militaires françaises à l'ensemble des festivités était systématique et, de ce point de vue, la cérémonie d'ouverture sur la place où se faisaient face le palais de la Résidence Générale et la cathédrale de Tunis fondus en un même ensemble par la foule des assistants était hautement symbolique, même si le Résident Fançois Manceron n'y prit point la parole. De ce point de vue, la laïcité républicaine est bien loin, et à plusieurs reprises les participants se félicitent discrètement de la bonne entente entre les autorités religieuses et civiles ${ }^{50}$. Certains même laissent apparaitre une position extrême, comme le député breton de Pontl'Abbé, Quéinnec, qui donne libre cours à l'expression de ses opinions après un repas non officiel:

Ce qui choque ici dans ce pays, ce qui déroute les indigènes, ce sont ces fameuses lois laïques, et le peu qu'on en importe en Tunisie est déjà trop. Je n'ai pas peur de le dire. Vive la Tunisie sous le régime chrétien des Francs, sous la loi catholique, celle de saint Louis et des croisés, celle du Cardinal Lavigerie et de Charles de Foucauld, et puisse ce Congrès donner une impulsion nouvelle à l'œuvre des Pères Blancs, et des multiples congrégations qui se sont donné la noble mission de rendre à l'Église romaine l'ancienne Numidie et toute l'Afrique proconsulaire ${ }^{51}$.

Si la tonalité générale et officielle est moins abrupte, préférant la notion de " croisade pacifique ", l'alliance conclue entre l'Église et le nationalisme français est néanmoins bien illustrée par une anecdote que rapporte l'abbé Mévellec:

Son Eminence (le cardinal Lépicier) a eu tout à l'heure un de ces gestes qui lui gagnent tous les cœurs. Auprès de sainte Perpétue, quelques soldats français traversaient la route avec le drapeau tricolore. Mgr Lépicier fait immédiatement stopper sa voiture, descend, et là, devant toute la foule, prend le drapeau français et baise l'étoffe en criant «Vive la France! » «Vive la France! » répond dans une formidable clameur la multitude des pèlerins... «Vive la France et vive le Pape ${ }^{52}$ !»

Les nations rivales ne s'y trompèrent d'ailleurs pas. Les Anglais se firent remarquer par la tiédeur de leur participation. Le correspondant britannique du P. Boubée, le P. Martindale lui écrit:

Je crains sans doute que je ne pourrai venir au congrès. De plus je crains qu'il n'y aura que très peu d'Anglais. Il me prendrait trop de temps de vous expliquer pourquoi... Vous me pardonnerez cette lettre si courte et si mal dactylographiée car j’ai horriblement sommeil et je ne puis ni parler ni penser. Soyez quand même sûr qu'à peu près toute

50 Voir, Actes, p. 512, les conclusions du P. PARRA, directeur national de L'apostolat de la prière en France.

51 MÉvelLeC, L'immortelle Carthage, p. 166.

52 Mévellec, L'immortelle Carthage, p. 184. 
l'Angleterre a entendu parler du Congrès de Sydney et en a vu des projections lumineuses. On ira en Irlande, c'est facile, mais je désire qu'on aille à ces congrès sans infection (si j’ose parler ainsi) de nationalisme ${ }^{53}$.

Les Italiens sont évidemment plus ouvertement amers et quelques sondages dans l'Unione, le journal fasciste de la communauté italienne de Tunisie sont significatifs: "Sur la terre où Rome a vaincu son plus grand ennemi, sur la terre où fleurirent les martyrs, les saints, et d'illustres docteurs de l'Église, les descendants les plus vrais, les plus légitimes de ces Romains et de ces chrétiens ont renouvelé devant vous (cardinaux et évêques d'Italie venus pour le Congrès) un pacte de fidélité qui les lie non plus à deux pouvoirs séparés et antagonistes, mais à une seule Rome régénérée, et aujourd'hui plus que jamais immortellement grande ${ }^{54}$. " Les prélats italiens avaient été beaucoup plus prudents dans leurs discours mais la mauvaise humeur du gouvernement italien se serait clairement manifestée s'il est vrai, comme le bruit en courut, que Mussolini avait refusé à un navire transportant des prélats français d'aborder à Naples pour que ses passagers viennent à la rencontre du Cardinal Lépicier partant pour le congrès 55 .

L'étude de l'expression et du jeu des nationalismes lors du congrès resterait à faire. Le thème du martyre qui le sous-tendait et la présence concomitante de tant de nationalités expliquent le nombre de discours relatant le martyrologe des nations, comme ceux des sections polonaise ou arménienne ${ }^{56}$.

Soulignons en conclusion que, dans un article intitulé "Après les fêtes de Carthage", L. Bertrand tirait ce bilan du congrès:

Ces fêtes ont été ce qu'elles devaient être: non seulement un grand acte de foi catholique, mais une belle manifestation de concorde internationale et, en même temps, un éloquent témoignage en faveur de l'œuvre française accomplie depuis cinquante ans par les nôtres en cette terre d'Afrique ${ }^{57}$.

Par œuvre, on entendait le développement des infrastructures tunisiennes, mais aussi la résurrection de l'Église d'Afrique voulue par Lavigerie et le triomphe de l'archéologie religieuse qui avait servi cette cause. Un triomphe international, certes, mais aussi le chant du cygne de cette archéologie religieuse. Le congrès, organisé sans tenir aucun compte de l'état de l'opinion, qu'il s'agisse des nationalistes tunisiens, des cercles religieux musulmans ou des tenants de la laïcité, avait profondément choqué de

Lettre du 12 février 1930 (Archives de la Prélature).

$54 \quad$ L'Unione du 14 mai 1930.

55 Mévellec, L'immortelle Carthage, p. 10; voir aussi la correspondance (avril 1930) du P. Boubée avec le P. Bacquet, de Rome, mortifié de voir que seuls les prêtres italiens bénéficient de billets à tarifs réduits financés par le gouvernement de la Péninsule.

56 Actes, p. 445-464 et 216-218.

$57 \quad$ La Tunisie française, 26 mai 1930. 
nombreux éléments de la société. Organisé à grands frais d'État, alors que la Tunisie souffrait lourdement des conséquences de la grande crise de 1929, il apparaissait comme une saignée financière injuste au seul profit de la catholicité. Le pouvoir beylical sortait discrédité de l'aventure par sa collaboration aux festivités du congrès. Enfin, ce dernier avait été l'occasion d'arrestations de jeunes manifestants nationalistes tunisiens. Ceux-là avaient été " généreusement " libérés, mais la Résidence Générale eut ensuite bien du mal à se dépêtrer de ce dossier embarrassant. Le bilan, une fois les fêtes passées, paraissait bien lourd. Il est significatif que le congrès soit expédié en deux lignes dans les éditions d'après-guerre de l'Histoire de la Tunisie d'Arthur Pellegrin ${ }^{58}$, significatif aussi qu'il ne soit même pas mentionné dans l'article "Carthage chrétienne" du Dictionnaire d'histoire et de géographie ecclésiastique, rédigé au même moment par les P.G. Lapeyre et Jean Ferron, successeurs du P. Delattre à la tête de l'archéologie chrétienne de Carthage ${ }^{59}$. La mort du P. Delattre un an et demi après le congrès, après cinquante-sept ans d'activité inlassable et militante à Carthage, scellait par ailleurs la fin d'une époque. Il n'y aura pas d'autre résurrection internationale de la Carthage antique par la suite, avant les grandes fouilles de l'Unesco qui rassemblèrent dans les années 1970, quarante ans après le congrès eucharistique, dix nations sur le site. Mais cette fois, c'était des Carthages punique, romaine et byzantine qu'il s'agissait, et pour faire pièce au delenda est Carthago du vieux Caton, ce n'était plus l'instauranda est Carthago (il faut rétablir, ressusciter, Carthage) du Cardinal Lavigerie qui était à l'ordre du jour, mais le servanda est Carthago (il faut sauver Carthage) de la communauté scientifique internationale. À chaque époque son mot d'ordre pour la vieille cité d'Afrique.

Jacques AlEXANDROPOUlOS

Université de Toulouse II

PLH-ERASME (EA 4153)

Pavillon de la Recherche

5, allées Antonio Machado

F-31058 Toulouse cedex 9

alexandropoulos.jacques@univ-tlse2.fr

A. Péllegrin, Histoire de la Tunisie des origines jusqu'à nos jours, Tunis, Namura, 1944, p. 175. 